\title{
Liver $\mathrm{CT}_{1}$ decreases following direct-acting antiviral therapy in patients with chronic hepatitis $C$ virus
}

\author{
Arjun N. A. Jayaswal ${ }^{1}(1) \cdot$ Christina Levick $^{1,2}$ (D) Jane Collier ${ }^{2} \cdot$ Elizabeth M. Tunnicliffe $^{1,3}$ (D) Matthew D. Kelly ${ }^{4}$. \\ Stefan Neubauer ${ }^{1,3} \cdot$ Eleanor Barnes $^{2,3,5}$ (1) $\cdot$ Michael Pavlides ${ }^{1,2,3}$ (])
}

Received: 27 August 2020 / Revised: 4 November 2020 / Accepted: 7 November 2020 / Published online: 28 November 2020

(c) The Author(s) 2020

\begin{abstract}
Purpose Direct-acting antiviral therapies (DAAs) for treatment of chronic hepatitis C virus (HCV) have excellent rates of viral eradication, but their effect on regression of liver fibrosis is unclear. The primary aim was to use magnetic resonance imaging (MRI) and spectroscopy (MRS) to evaluate changes in liver fibrosis, liver fat and liver iron content (LIC) in patients with chronic HCV following treatment with DAAs.

Methods In this prospective study, 15 patients with chronic HCV due to start treatment with DAAs and with transient elastography (TE) $>8 \mathrm{kPa}$ were recruited consecutively. Patients underwent MRI and MRS at baseline (before treatment), and at 24 weeks and 48 weeks after the end of treatment (EoT) for the measurement of liver $\mathrm{cT}_{1}$ (fibroinflammation), liver fat and $\mathrm{T}_{2} *$ (LIC).

Results All patients achieved a sustained virological response. Liver $\mathrm{cT}_{1}$ showed significant decreases from baseline to 24 weeks post EoT ( 876 vs $806 \mathrm{~ms}, p=0.002, n=15$ ), baseline to 48 weeks post EoT ( 876 vs $788 \mathrm{~ms}, p=0.0002, n=13$ ) and 24 weeks post EoT to 48 weeks post EoT ( 806 vs $788 \mathrm{~ms}, p=0.016, n=13$ ). Between baseline and 48 weeks EoT significant reduction in liver fat $(5.17 \%$ vs $2.65 \%, p=0.027)$ and an increase in reported LIC (0.913 vs $0.950 \mathrm{mg} / \mathrm{g}, p=0.021)$ was observed.

Conclusion Liver $\mathrm{cT}_{1}$ decreases in patients with chronic $\mathrm{HCV}$ undergoing successful DAA treatment. The relatively fast reduction in $\mathrm{cT}_{1}$ suggests a reduction in inflammation rather than regression of fibrosis.
\end{abstract}

Keywords $\mathrm{T}_{1}$ mapping $\cdot$ Iron corrected $\mathrm{T}_{1} \cdot$ Direct-acting antivirals $\cdot$ Hepatitis $\mathrm{C}$ virus

Eleanor Barnes and Michael Pavlides are Joint senior Authors.

Electronic supplementary material The online version of this article (https://doi.org/10.1007/s00261-020-02860-5) contains supplementary material, which is available to authorized users.

Michael Pavlides

michael.pavlides@cardiov.ox.ac.uk

1 Oxford Centre for Clinical Magnetic Resonance Research, Division of Cardiovascular Medicine, Radcliffe Department of Medicine, University of Oxford, Oxford, UK

2 Translational Gastroenterology Unit, University of Oxford, Oxford, UK

3 Oxford NIHR Biomedical Research Centre, Oxford, UK

4 Perspectum Ltd, Oxford, UK

5 Peter Medawar Building for Pathogen Research, University of Oxford, Oxford, UK

\section{Introduction}

Chronic hepatitis $\mathrm{C}$ virus (HCV) affects an estimated 71 million people worldwide [1] and is associated with the development of liver cirrhosis, hepatocellular carcinoma (HCC), liver failure and death. Direct-acting antiviral therapies (DAAs) have high rates of viral eradication $(>95 \%)$ and have transformed the treatment of HCV [2-12].

Currently, even after achieving sustained virological response (SVR), patients with HCV liver cirrhosis are recommended for HCC surveillance by ultrasound combined with alpha-fetoprotein (AFP) every 6 months indefinitely [13]. However, the risk factors for development of HCC post SVR are not well defined. Recent evidence suggests that sustained virological response (SVR) reduces the risk HCC, albeit not removing the risk completely [14-16]. Fibrosis-4 (FIB-4) score $>3.25$ and models involving age, platelet count, serum aspartate aminotransferase/alanine 
aminotransferase (AST/ALT) ratio and albumin have been identified as predictors of HCC post SVR [17, 18].

There is a growing body of evidence showing a reduction in non-invasive biomarkers of fibrosis in significant proportions of patients following DAA treatment [19-26]. However, it is unclear whether these changes are due to regression of inflammation or fibrosis, or both. In a study of paired liver biopsy pre and post DAA therapy, the maintenance or regression of fibrosis and inflammation varied across patients, while non-invasive markers overestimated histological regression of fibrosis [27]. Furthermore, it is unclear whether changes in fibroinflammation reduce risk of HCC development.

Liver fibrosis and inflammation increases the liver's extracellular water content, which can be quantified by the magnetic resonance imaging (MRI) parameter $\mathrm{T}_{1}$, when corrected for iron content [28] $\left(\mathrm{cT}_{1}\right)$. Liver $\mathrm{cT}_{1}$ correlates with liver fibrosis, inflammation and ballooning [29, 30], has excellent repeatability and reproducibility [31] and can predict clinical outcomes [32]. In the same scan $\mathrm{T}_{2}{ }^{*}$ can quantify liver iron content (LIC [33]) and proton magnetic resonance spectroscopy (1H-MRS) can quantify liver fat content, having been validated extensively against histology [34]. Liver $\mathrm{cT}_{1}$ has been used to assess treatment response in patients with non-alcoholic steatohepatitis (NASH) [35] and therefore could be used to assess change in the liver parenchyma after HCV cure by DAAs.

The primary aim of this study was to evaluate changes in liver $\mathrm{cT}_{1}$ (fibroinflammation), $\mathrm{T}_{2}{ }^{*}$ (LIC) and ${ }^{1} \mathrm{H}$-MRS (liver fat) in patients with chronic HCV following treatment with DAAs. Secondary aims were to assess changes in other noninvasive biomarkers of liver disease including transient elastography (TE), simple blood tests and serum-based fibrosis scores.

\section{Patients and methods}

In this single center, prospective, observational cohort study patients with chronic HCV were consecutively invited from hepatology outpatient clinics at the John Radcliffe Hospital, Oxford, UK, between December 2014 and September 2017. Chronic HCV was defined by positive HCV RNA assays on more than two occasions 6 months apart. A sustained virological response (SVR) was defined as a negative HCV RNA 12 weeks post DAA treatment. All patients with a most recent clinical TE measurement $>8 \mathrm{kPa}$ who were approved to start treatment with DAAs by a multi-disciplinary team were invited to take part in the study. Exclusion criteria were contraindications to MRI. Patients were treated with the DAA treatment regimens prescribed by their clinical care team, which were not affected by participation in the study.
Patients underwent study assessments comprising MRI, TE and blood sampling at baseline (before treatment), and a 24 weeks and 48 weeks after the end of treatment (EoT). The study was approved by the local Research Ethics Committee (ref no: 13/SC/0234) and conforms to the declaration of Helsinki. All patients gave written, informed consent to take part.

\section{MRI protocol}

All MRI scans were carried out at the University of Oxford Centre for Clinical Magnetic Resonance Research (OCMR) on a 3T Siemens Tim Trio scanner (Erlangen, Germany). Patients underwent the LiverMultiScan ${ }^{\mathrm{TM}}$ (Perspectum Diagnostics Ltd, Oxford, UK) acquisition protocol for $\mathrm{T}_{1}$ and $\mathrm{T}_{2}{ }^{*}$ mapping and ${ }^{1} \mathrm{H}-\mathrm{MRS}$, after fasting for at least $4 \mathrm{~h}$, as described previously [28, 29].

\section{MRI analysis}

$\mathrm{T}_{1}$ and $\mathrm{T}_{2} *$ maps were analyzed using LiverMultiScan ${ }^{\mathrm{TM}}$ software (Perspectum Ltd, Oxford UK) for the purpose of generating and analyzing $\mathrm{cT}_{1}$ maps by the following steps:

(1) Three regions of interest (ROIs) were placed manually on $\mathrm{T}_{2} *$ color maps to obtain a representative LIC (estimated in units of $\mathrm{mg} / \mathrm{g}$ dry weight).

(2) This LIC was fed into an algorithm that transforms the measured $\mathrm{T}_{1}$ map into a $\mathrm{cT}_{1}$ map in such a way that corrects for any underestimation of $\mathrm{T}_{1}$ caused by increased LIC [28].

(3) On the $\mathrm{cT}_{1}$ map, the liver was segmented semi-automatically, excluding the blood vessels, large bile ducts and other abnormalities (e.g., cysts). The mean value of $\mathrm{cT}_{1}$ within the liver was calculated and taken as the final $\mathrm{cT}_{1}$ value.

In the derivation of $\mathrm{T}_{1}$ and $\mathrm{T}_{2}{ }^{*}$ maps, $\mathrm{R}^{2}$ maps are generated, which are a measure of the goodness of fit of the MR signal intensity to $\mathrm{T}_{1}$ and $\mathrm{T}_{2}{ }^{*}$ relaxation curves. When placing ROIs in the $\mathrm{T}_{1}$ and $\mathrm{T}_{2}{ }^{*}$ maps, mean $R^{2}$ values were also calculated. $\mathrm{T}_{2}{ }^{*}$ map $R^{2}$ values had to be $>0.95$, and $\mathrm{T}_{1}$ map $R^{2}$ values had to be $>0.99$ for all liver measurements to be considered valid.

${ }^{1} \mathrm{H}$-MRS acquisitions were combined and fitted using the OXSA toolbox [36] implementation of the AMARES algorithm [37] with an in-house MATLAB (The Mathworks, Natick, MA, USA) script [38]. Liver fat was expressed as a ratio of the total fat signal divided by the total water + fat signal $(\%)$. 


\section{Transient elastography}

TE (Fibroscan®, Echosens, Paris, France) was performed by a trained professional, and a valid reading was defined according to the manufacturer's criteria (10 valid shots and $\mathrm{IQR} /$ median ratio $<0.3$ ). TE was performed on the same day as the MRI scan and the blood tests where possible, alternatively the most recent clinically performed value was used (maximum 1 weeks' interval).

\section{Blood sampling}

Blood samples were drawn on the same day as the MRI scan. Simple scores for serum-based fibrosis markers FIB-4 [39], aspartate aminotransferase to platelet ratio index (APRI) [40] and AST/ALT ratio were calculated.

\section{Clinical data and anthropometric measures}

Diabetes status, alcohol consumption, evidence of decompensation, height, weight, and body mass index (BMI) measurements were recorded. Patients consuming more than 14units of alcohol per week were classed as consuming alcohol above the recommended limits. Patients with cirrhosis were defined by Ishak fibrosis stage $\geq$ F5 on a clinically indicated biopsy or having clinical decompensation at baseline assessment.

\section{Patient follow-up}

Patients were also followed up for the development/recurrence of HCC and new liver decompensation events (ascites, variceal bleeding, hepatic encephalopathy, liver transplantation and liver-related mortality) from recruitment until the last study visit of the last patient in the study by review of their electronic patient records.

\section{Statistical analysis}

Median and interquartile ranges (IQR) were reported for patient characteristics due to the small numbers involved. The primary outcome was the change in liver $\mathrm{cT}_{1}$ following DAA treatment. Secondary outcomes included changes in liver fat, LIC, liver stiffness (measured by TE), FIB-4, APRI and AST/ALT scores and other blood-based biomarkers. Paired comparisons of these parameters between study timepoints was performed using the non-parametric paired Wilcoxon test.

Temporal changes in liver $\mathrm{cT}_{1}$ were compared by Wilcoxon rank test between patients with and without cirrhosis. Correlations between liver $\mathrm{cT}_{1}$ and $\mathrm{TE}$ and the influence of liver fat on liver $\mathrm{cT}_{1}$ [41] were investigated using the Spearman's rank correlation coefficient. We did not correct for multiple comparisons as there were small numbers of patients, this being a proof-of-principle study. Significance level for all statistical tests was set at 0.05. All statistical analysis was performed, and all plots were generated using $\mathrm{R}$ statistical software [42].

\section{Results}

Seventeen patients were recruited and assessed at baseline. One patient was excluded due to their inability to hold their breath during the MRI scan and one patient was lost to follow-up after their baseline scan. Fifteen patients returned for follow-up visits at 24 weeks EoT and were included in analysis, of which 2 were lost to follow-up at 48 weeks EoT (Fig. 1). All patients achieved SVR as confirmed by undetectable HCV RNA assay 24 weeks after treatment.

\section{Patient characteristics}

The fifteen patients included in the analysis had median (IQR) age 58 (52-60), median (IQR) BMI 25.7 (23.2-28.4) $\mathrm{kg} / \mathrm{m}^{2}$ and $10(75 \%)$ were male. Seven $(47 \%)$ patients had genotype 1 (GT1) infection, three (20\%) had GT2 and five (33\%) had GT3. Four (27\%) patients self-reported alcohol consumption exceeding the recommended limits and seven (47\%) had cirrhosis at baseline. Individual patients' details are reported in Supplementary Table 1.

\section{Liver $\mathrm{cT}_{1}$}

Liver $\mathrm{cT}_{1}$ showed a significant decrease from baseline to 24 weeks EoT (876 vs 806 ms, $p=0.002, n=15$, Fig. 2a), from baseline to 48 weeks EoT ( 876 vs $788 \mathrm{~ms}, p=0.0002$, $n=13$ ) and from 24 weeks EoT to 48 weeks EoT (806 vs $788 \mathrm{~ms}, p=0.016, n=13$, Table 1, Fig. 2a). Example images at baseline and 24 weeks EoT are shown in Fig. 3.

\section{Liver fat}

Liver fat measurements were available for 12 patients at baseline and 24 weeks EoT and in 11 patients at 24 and 48 weeks EoT. Median liver fat showed a trend towards decrease from baseline to 24 weeks EoT (5.17\% vs $2.54 \%$, $p=0.064, n=12$ ) reaching a significant decrease between baseline and 48 weeks EoT $(5.17 \%$ vs $2.65 \%, p=0.027$, $n=11$ ). There was no correlation between change in liver fat and change in $\mathrm{cT}_{1}$.

\section{Liver iron}

LIC increased between baseline and 24 weeks EoT (0.913 vs $1.019 \mathrm{mg} / \mathrm{g}$ dry weight, $p=0.022, n=15$ ) and between 
Fig. 1 Study flow diagram. EoT end of treatment

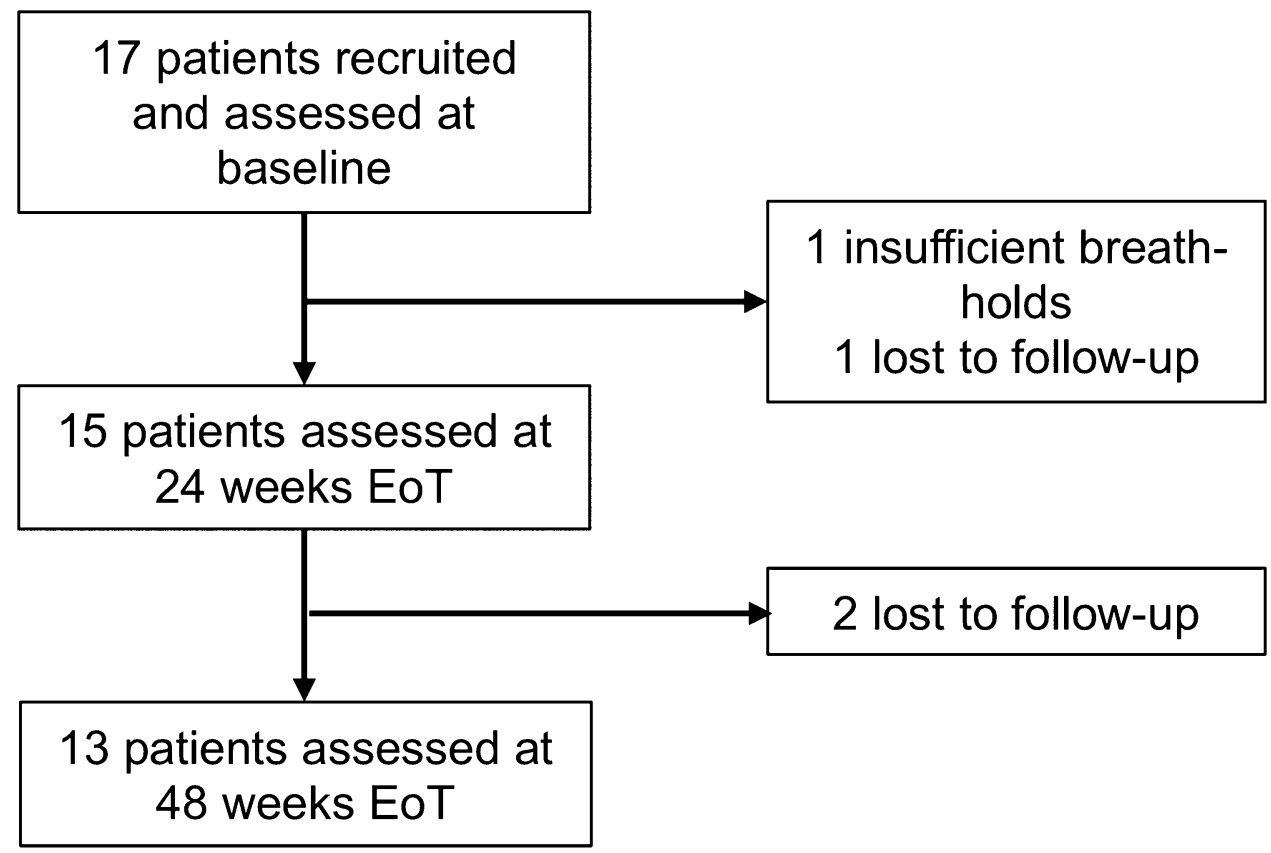

Table 1 Patient demographics at baseline, 24 and 48 weeks after the endo of treatment (EoT)

\begin{tabular}{|c|c|c|c|c|}
\hline & $\begin{array}{l}\text { Baseline }(n=15) \\
\text { median }(\mathrm{IQR})\end{array}$ & $\begin{array}{l}24 \text { weeks EoT }(n=15) \\
\text { median }(\mathrm{IQR})\end{array}$ & $\begin{array}{l}48 \text { weeks EoT }(n=13) \\
\text { median (IQR) }\end{array}$ & $\begin{array}{l}p \text { value } \\
\text { (baseline vs } \\
48 \text { weeks) }\end{array}$ \\
\hline BMI $\left(\mathrm{kg} / \mathrm{m}^{2}\right)$ & $25.7(23.2-28.4)$ & $26.4(23.8-27.5)$ & $26.6(24.3-30.7)$ & \\
\hline Bilirubin (mmol/L) & $12(8-16)$ & $12(10-18)$ & $9(9-13)$ & 0.0658 \\
\hline ALT (IU/L) & $120(59-134)$ & $28(21-34)$ & $23(17-29)$ & $<0.001$ \\
\hline ALP (IU/L) & $96(70-152)$ & $71(60-105)$ & 77 (64-93) & 0.0137 \\
\hline Albumin (g/L) & $36(34-37)$ & $39(37-40)$ & $38(36-39)$ & 0.0172 \\
\hline GGT (IU/L) & $107(88-135)$ & $36(24-71)$ & $33(24-73)$ & $<0.001$ \\
\hline AST (IU/L) & $94(67-140)$ & $32(29-42)$ & $29(25-35)$ & 0.0078 \\
\hline Ferritin $(\mu \mathrm{g} / \mathrm{l})$ & $184(42-531)$ & $57(10-169)$ & $92(57-184)$ & 0.156 \\
\hline Transferrin saturation (\%) & $35(17-43)$ & $31(17-44)$ & $33(26-40)$ & 0.751 \\
\hline Trigs (mmol/L) & $0.99(0.70-1.64)$ & $0.78(0.62-1.00)$ & $0.70(0.61-0.97)$ & 0.0840 \\
\hline $\mathrm{HDL}(\mathrm{mmol} / \mathrm{L})$ & $1.1(0.9-1.4)$ & $1.4(1.2-1.6)$ & $1.5(1.1-1.6)$ & 0.0453 \\
\hline LDL (mmol/L) & $2.5(2.2-2.8)$ & $2.5(2.3-2.9)$ & $2.5(2.3-2.7)$ & 0.8885 \\
\hline Total:HDL ratio & $4.22(3.07-5.00)$ & $2.90(2.60-4.03)$ & $2.94(2.71-3.63)$ & 0.0098 \\
\hline Platelets $\left(\times 10^{9} / \mathrm{L}\right)$ & $192(126-239)$ & $223(127-233)$ & $233(113-286)$ & 0.0661 \\
\hline \multicolumn{5}{|c|}{ Non-invasive scores of liver disease severity } \\
\hline Child-Pugh score & $6(5-6)$ & $5(5-5)$ & $5(5-5)$ & 0.3458 \\
\hline FIB-4 & $2.51(1.96-4.48)$ & $2.27(1.57-3.73)$ & $1.39(1.16-4.06)$ & 0.0156 \\
\hline AST/ALT & $1.158(0.703-1.196)$ & $1.400(1.160-1.467)$ & $1.29(1.052-1.771)$ & 0.0781 \\
\hline APRI & $1.185(0.776-2.299)$ & $0.406(0.259-0.541)$ & $0.219(0.181-0.658)$ & 0.0156 \\
\hline $\mathrm{TE}(\mathrm{kPa})$ & $19.5(8.2-24.3)$ & $11.9(7.4-19.2)$ & $10.1(7.4-18.7)$ & 0.0020 \\
\hline
\end{tabular}

$A L T$ alanine aminotransferase, $A L P$ alkaline phosphatase, $A S T$ aspartate aminotransferase, $G G T$ gamma-glutamyl transferase, Trigs triglycerides, $H D L$ high density lipoprotein, $L D L$ low density lipoproteins, FIB-4 fibrosis-4, APRI AST to platelet ratio index, FIB-4 fibrosis - 4, AST/ALT score aspartate aminotransferase/alanine aminotransferase ratio, $A P R I$ aspartate aminotransferase to platelet ratio index, $T E$ transient elastography 

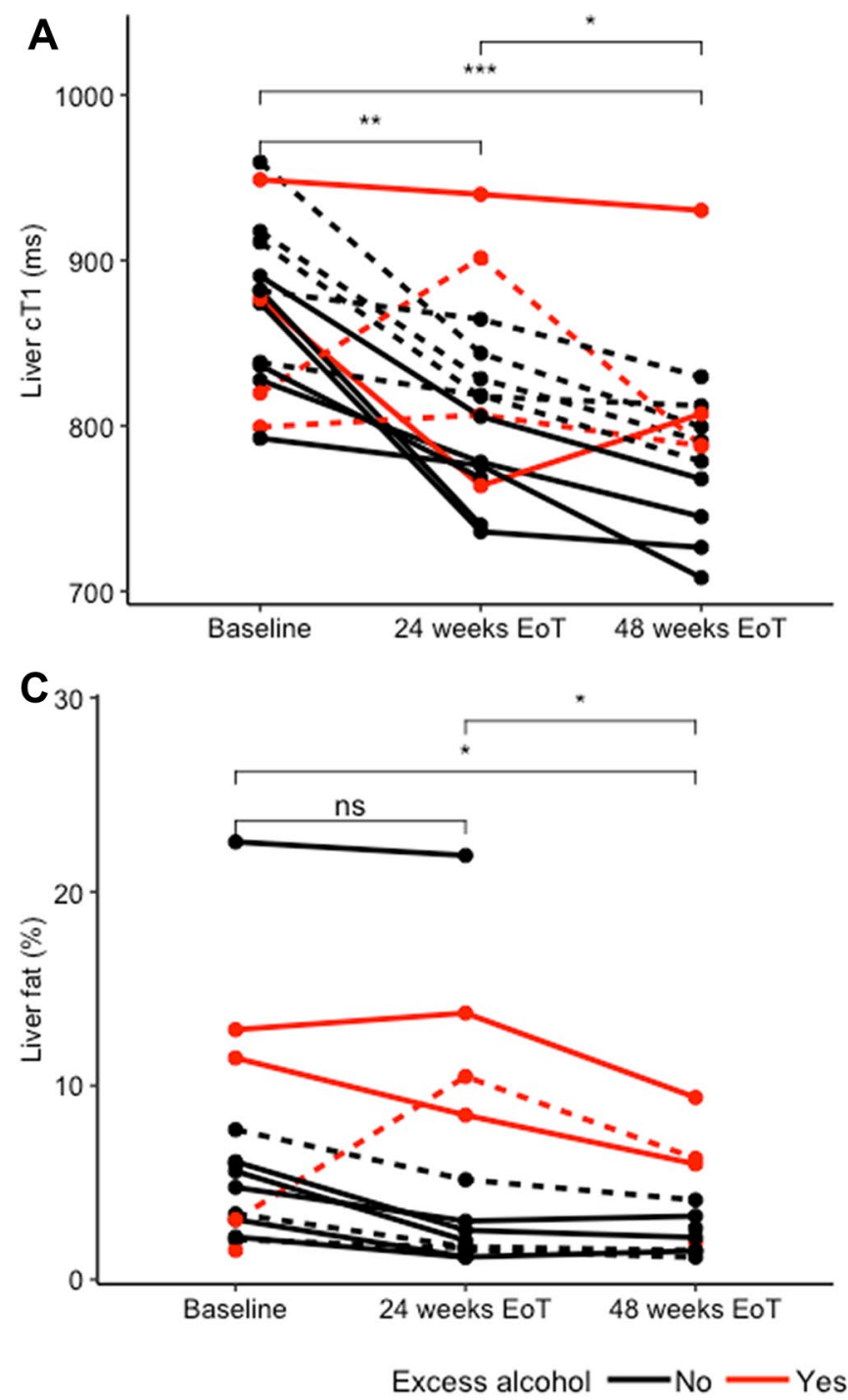

Fig. 2 Response in non-invasive markers of liver disease to DAA treatment from baseline to 24 weeks EoT, and 48 weeks EoT. Plots show response to treatment by a liver $\mathrm{cT}_{1}, \mathbf{b}$ transient elastography

baseline and 48 weeks EoT ( 0.913 vs $0.950 \mathrm{mg} / \mathrm{g}, p=0.021$, $n=13$ ), but there was no significant change between 24 and 48 weeks EoT ( $p=0.470$, Table 1, Fig. 2d).

\section{Transient elastography}

TE showed significant decreases between baseline and 24 weeks EoT ( 19.5 vs $11.9 \mathrm{kPa}, p=0.005, n=12$ ), between baseline and 48 weeks EoT (19.5 vs $10.1 \mathrm{kPa}, p=0.005$, $n=10)$ and between 24 and 48 weeks EoT (11.9 vs $10.1 \mathrm{kPa}$, $p=0.041, n=12$, Table 2, Fig. 2b).

TE measurements were significantly correlated with liver $\mathrm{cT}_{1}(r=0.60, p<0.0001, n=39$, Supplementary Figure S1) with a moderate correlation observed in pretreatment measurements $(r=0.547, p=0.05, n=12)$ and a
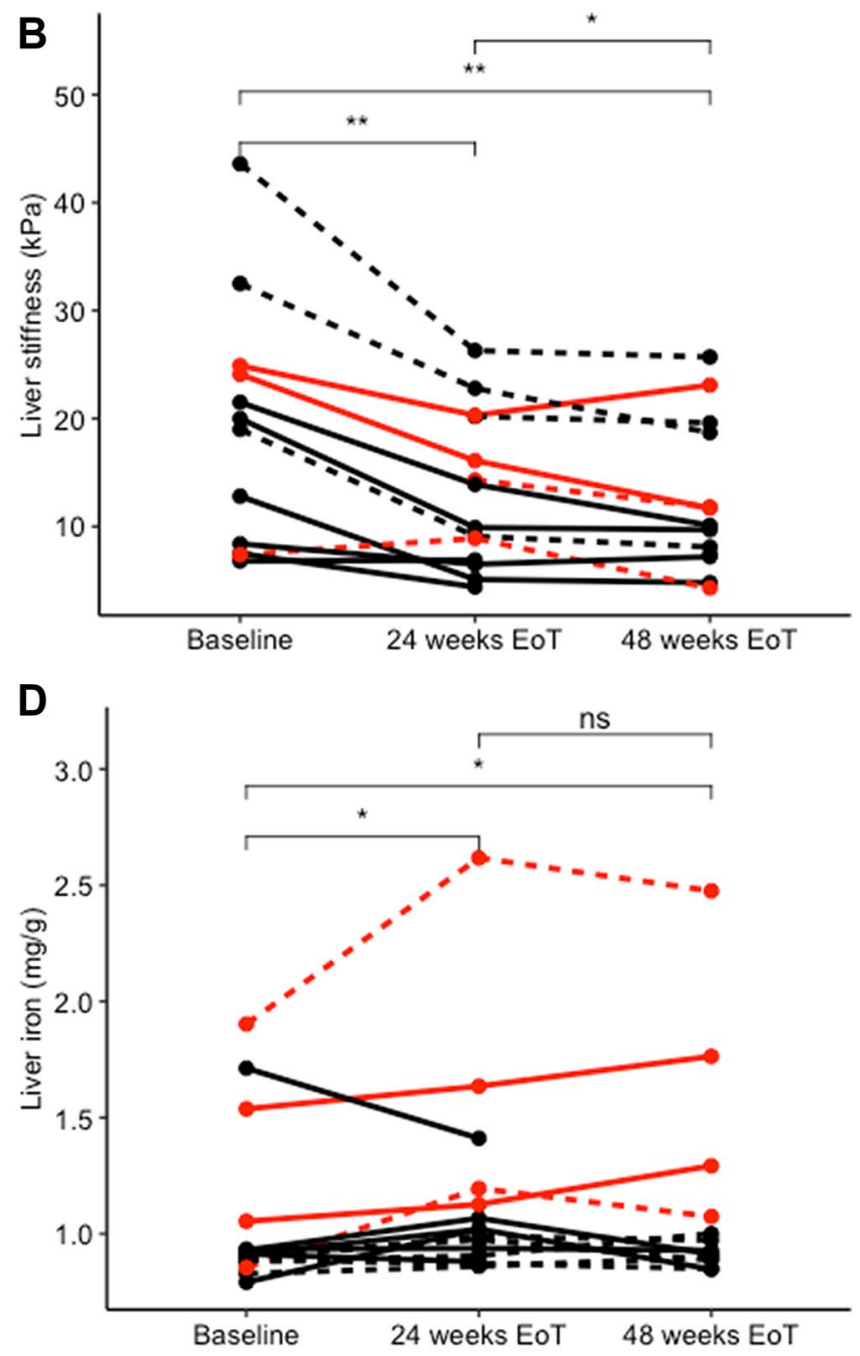

Cirrhosis - No - Yes

(TE), c liver fat, $\mathbf{d}$ liver iron content. Each line represents an individual patient. * denotes $p<0.05$, ** denotes $p<0.01$, ns denotes $p>0.05$. EoT end of treatment, $D A A$ direct-acting antiviral therapy

weaker correlation observed in post-treatment measurements $(r=0.134, p=0.003, n=27)$. Reductions in $\mathrm{cT}_{1}$ correlated moderately $(r=0.500, p=0.015, n=23)$ with reductions in TE.

\section{Blood-based biomarkers}

From both baseline to 24 weeks EoT and baseline to 48 weeks EoT, significant reductions were observed in ALT $(p<0.001$ and $p<0.001)$, AST $(p=0.004$ and $p=0.008)$, ALP $(p=0.014$ and $p=0.014)$, GGT $(p<0.001$ and $p<0.001)$, FIB-4 $(p=0.023$ and $p=0.016)$, APRI $(p=0.008$ and $p=0.016)$, and significant increases were observed in albumin $(p=0.005$ and $p=0.017)$ and HDL $(p=0.028$ and $p=0.045)$. 

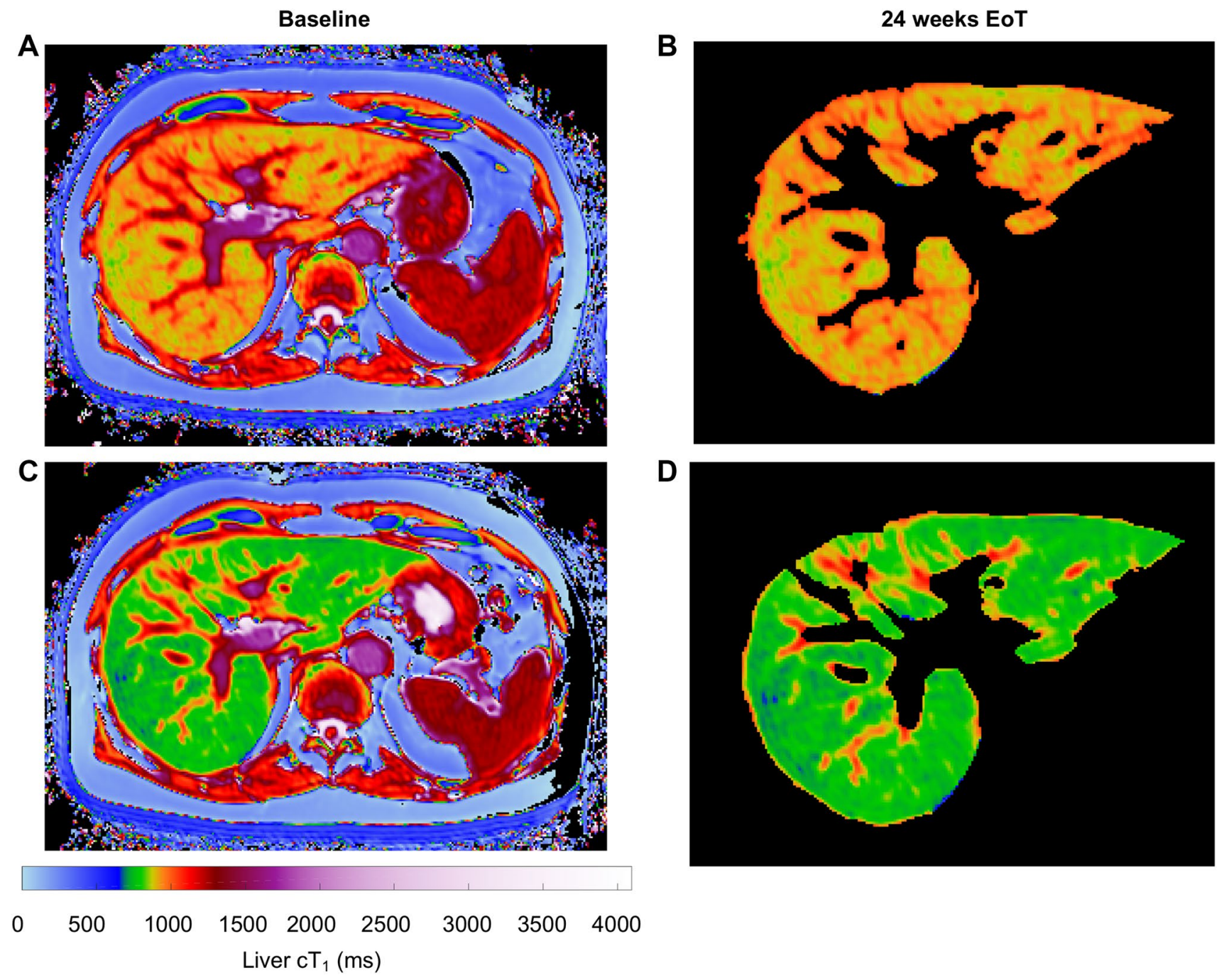

Fig. 3 Abdominal $\mathrm{cT}_{1}$ images and segmentation masks pre and post treatment. Abdominal $\mathrm{cT}_{1}$ images are shown from a patient with chronic hepatitis $\mathrm{C}$ virus at baseline (a) and at 24 weeks after end of treatment (EoT) (b), together with the semi-automatically segmented

liver $\mathrm{cT}_{1}$ masks from the same patient at baseline (c) and 24 weeks EoT (d). Mean liver $\mathrm{cT}_{1}$ at baseline was $874 \mathrm{~ms}$ and 24 weeks EoT was $735 \mathrm{~ms}$
Table 2 Magnetic resonance parameters at baseline, and 24 and 48 weeks after the end of treatment (EoT)

\begin{tabular}{llllc}
\hline & $\begin{array}{l}\text { Baseline }(n=15) \\
\text { median (IQR) }\end{array}$ & $\begin{array}{l}\text { 24 weeks EoT }(n=15) \\
\text { median (IQR) }\end{array}$ & $\begin{array}{l}48 \text { weeks EoT } \\
(n=13) \text { median } \\
(\mathrm{IQR})\end{array}$ & $\begin{array}{l}p \text { value } \\
\text { (baseline vs } \\
48 \text { weeks) }\end{array}$ \\
\hline Liver cT $_{1}(\mathrm{~ms})$ & $876(832-901)$ & $806(772-836)$ & $788(767-807)$ & $<\mathbf{0 . 0 0 1}$ \\
Liver fat $(\%)$ & $5.17(2.64-6.03)$ & $2.54(1.63-8.48)$ & $2.65(1.71-5.03)$ & $\mathbf{0 . 0 2 7}$ \\
Liver iron $(\mathrm{mg} / \mathrm{g})$ & $0.913(0.884-0.994)$ & $1.019(0.922-1.161)$ & $0.950(0.895-1.129)$ & $\mathbf{0 . 0 2 1}$ \\
\hline
\end{tabular}

From baseline to 24 weeks EoT significant reductions were observed in AST/ALT $(p=0.027)$ and ferritin $(p=0.023)$. From baseline to 48 weeks EoT significant reductions were observed in cholesterol:HDL ratio $(p=0.010)$.
Between 24 and 48 weeks EoT, AST, ALT, GGT and APRI showed trends towards decrease $(p=0.059, n=11$; $p=0.077, n=12 ; p=0.076, n=13$ and $p=0.064, n=10$, respectively) but no significant reductions were observed. 
A significant increase in platelet count was observed $(p=0.041, n=10)$.

\section{Patient follow-up}

No de novo occurrences of HCC or recurrences of HCC were observed following DAA treatment.

\section{Influence of cirrhosis on liver $\mathrm{cT}_{1}$}

Seven patients had clinical or histologically diagnosed cirrhosis. There was no significant difference in mean baseline $\mathrm{cT}_{1}$ between patients with and without cirrhosis (863 vs $866 \mathrm{~ms}, p=0.694)$. Liver $\mathrm{cT}_{1}$ was significantly higher in the group with than without cirrhosis at 24 weeks EoT (840 ms vs $789 \mathrm{~ms}, p=0.014$, Fig. 4). Between baseline and 24 weeks EoT there was a significant drop in $\mathrm{cT}_{1}$ in patients without cirrhosis ( 859 vs $767 \mathrm{~ms}, p=0.008$ ) but not in patients with cirrhosis ( $863 \mathrm{vs} 827 \mathrm{~ms}, p=0.375$ ). There was no significant difference in mean $\mathrm{cT}_{1}$ at 48 weeks between patients with and without cirrhosis $(p=0.234)$ nor any difference in magnitude of reduction in $\mathrm{cT}_{1}$ between 24 and 48 weeks EoT. Both groups were overall significantly reduced at 48 weeks EoT from baseline ( $p=0.031$ for both).

\section{Effects of alcohol consumption on non-invasive markers of liver disease}

Patients with self-reported excess alcohol consumption throughout the study $(n=4)$ had significantly higher levels of LIC ( $1.55 \mathrm{mg} / \mathrm{g}$ vs $0.96 \mathrm{mg} / \mathrm{g}$ dry weight, $p<0.001)$, liver fat $(9.19 \%$ vs $3.91 \%, p=0.004)$ and GGT levels $(133$

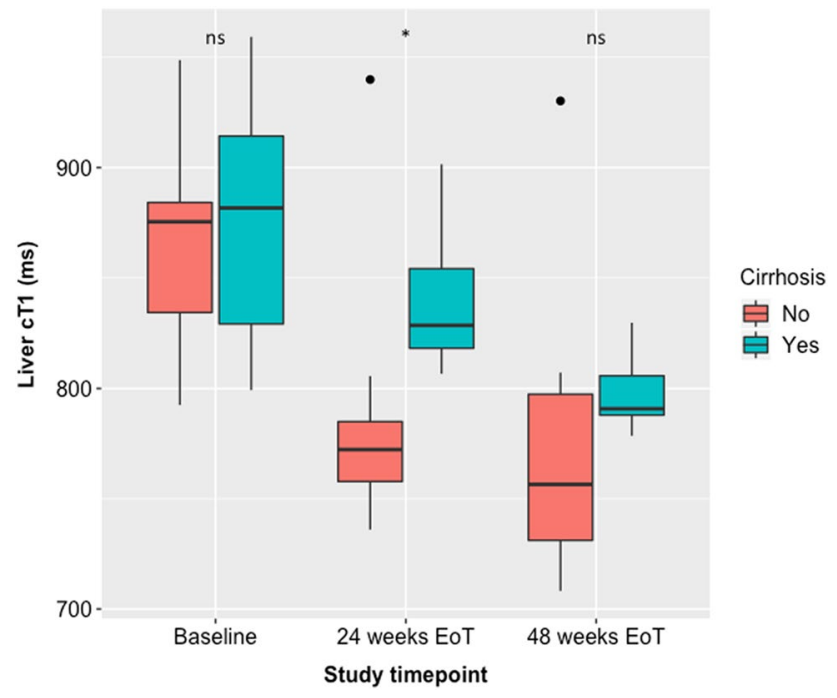

Fig. 4 Comparison of liver $\mathrm{cT}_{1}$ between patients with and without cirrhosis at baseline, 24 weeks EoT and 48 weeks EoT. EoT end of treatment, $*$ denotes $p<0.05$. Dots represent outliers vs $59 \mathrm{IU} / \mathrm{L}, p=0.011)$ than those without excess alcohol consumption.

When these patients were excluded from the analysis, liver $\mathrm{cT}_{1}$ showed a significant decrease from baseline to 24 weeks EoT ( 874 vs $798 \mathrm{~ms}, p<0.001, n=11$ ), from baseline to 48 weeks EoT ( 877 vs $773 \mathrm{~ms}, p=0.004, n=9$ ) and from 24 to 48 weeks EoT ( 808 vs 773, $p=0.004, n=9$ ). Liver fat showed a significant decrease from baseline to 24 weeks EoT ( 6.38 vs $4.47 \%, p=0.004, n=9$ ), from baseline to 48 weeks EoT ( 5.17 vs $2.53 \%, p=0.031, n=6$ ), but not from 24 to 48 weeks EoT ( $p=0.313)$. TE showed a significant decrease from baseline to 24 weeks EoT (19.1 vs $11.7 \mathrm{kPa}, p=0.008, n=9)$, baseline to 48 weeks EoT ( 22.5 vs $12.0 \mathrm{kPa}, p=0.016, n=7$ ) and showed a trend towards decrease from 24 to 48 weeks EoT ( $p=0.08, n=8$ ). LIC did not change between any timepoints when excluding those patients who consumed excess alcohol.

\section{Discussion}

In this study, liver $\mathrm{cT}_{1}$ has been used for the first time to track liver fibroinflammatory response to DAA treatment of chronic $\mathrm{HCV}$. The reductions in liver $\mathrm{cT}_{1}$ from baseline to 24 and 48 weeks EoT suggest both short and medium-term improvements in liver fibroinflammatory disease with $\mathrm{HCV}$ treatment.

Our results are consistent with a similar sized study by Bradley et al. $(n=17)$ at $1.5 \mathrm{~T}$, in which early reductions in liver $\mathrm{T}_{1}$ of $35 \mathrm{~ms}$ were observed after DAA-achieved SVR [19]. Our reductions of $70 \mathrm{~ms}$ in liver $\mathrm{cT}_{1}$ at $3 \mathrm{~T}$ by 24 weeks EoT were twice as much as Bradley's result. This is to be expected as the magnitude of liver $\mathrm{T}_{1}$ increases (almost linearly) with magnetic field strength. This reduction in $\mathrm{cT}_{1}$ is clinically meaningful, being nearly two times the $\mathrm{cT}_{1}$ repeatability coefficient and comparable to reductions in $\mathrm{cT}_{1}$ observed in a study investigating response to treatment for non-alcoholic steatohepatitis (NASH) [31, 35, 43].

In our study, as the reductions in $\mathrm{cT}_{1}$ between 24 and 48 weeks EoT were smaller than the reductions between baseline and 24 weeks EoT, most of the reduction in $\mathrm{cT}_{1}$ was likely due to resolving inflammation rather than fibrosis as liver fibrosis reversal has shown to be a slow process [44]. In contrast, the reduction in liver stiffness between baseline and 24 weeks EoT, but not between 24 and 48 weeks EoT conflicts with the assumption that TE is correlated only with fibrosis, but that inflammation is significantly contributing to the TE signal.

Liver fat and cholesterol:HDL ratio reduced significantly between baseline and 48 weeks EoT. Hepatic lipid accumulation is associated with chronic HCV and steatosis has been shown to be an independent risk-factor for HCC development with chronic HCV-related cirrhosis [45]. Reduction in 
liver fat may therefore also improve patients' outcomes, but a larger cohort with longer follow-up than our study would be required to investigate this. Our results suggest that $\mathrm{HCV}$ cure by DAA treatment may reduce liver fat regardless.

A small but statistically significant increase in LIC was observed from baseline to 48 weeks EoT. The reported LIC is a surrogate marker, calculated from and inversely proportional to the $\mathrm{T}_{2}{ }^{*}$ relaxation time. $\mathrm{T}_{2} *$ itself is a composite of the $\mathrm{T}_{2}$ relaxation time and magnetic field inhomogeneity. It is likely that liver tissue $\mathrm{T}_{2}$ has fallen (as observed by Bradley et al. [19]) with improvement in liver fibroinflammation, causing a decrease in $\mathrm{T}_{2}{ }^{*}$ and increase in reported LIC in turn, rather than an increase in actual tissue iron. An increase in LIC was observed in each patient who consumed excess alcohol (Fig. 2d), but no significant change was observed in patients who did not. This indicates that the increase in LIC is driven solely by excess alcohol and suggests that when monitoring these patients following treatment, one should not assume that a small drop in $\mathrm{T}_{2} *$ necessarily equates to an increase in tissue iron. Serum ferritin levels have been shown to fall in patients following viral eradication [46]. This could mean that after treatment the liver regains more capacity to store iron and takes up excess iron circulating in serum. Alternatively, a decrease in ferritin could be a response to reduction in inflammation, secondary to viral eradication. The latter scenario could explain our findings of increases in $\mathrm{T}_{2}{ }^{*}$-derived LIC and the observed trend towards decrease in ferritin but maintenance of transferrin saturations. Paired iron profiles were not available in all patients which may be the reason for the reduction in ferritin not reaching significance.

Patients drinking excess alcohol had higher liver fat and LIC across all timepoints in the study. Higher histological liver iron has also previously been observed in patients with both alcohol related liver disease (ArLD) and HCV than with HCV alone [47]. The reference value for elevated LIC is $1.7 \mathrm{mg} / \mathrm{g}$ [48] and our patients were at the higher end of the normal spectrum. It is known that patients with ArLD frequently present with evidence of high ferritin and/or iron overload, and even moderate alcohol consumption can increase markers of iron storage [49]. The only participants whose liver $\mathrm{cT}_{1}$ did not decrease between each time point were those that had been drinking heavily. Alcohol causes ongoing inflammation and fibrosis, which in continued alcohol abuse is a persistent upwards trajectory. Although HCV cure may reduce the contribution of HCV to inflammation and fibrosis, it may not be sufficient to reduce liver $\mathrm{cT}_{1}$ overall in these patients.

Patients without cirrhosis showed a greater initial decrease in liver $\mathrm{cT}_{1}$ than those with cirrhosis. We have also shown that liver $\mathrm{cT}_{1}$ continues to decrease between 24 and 48 weeks EoT, albeit at a lower rate than between baseline and 24 weeks EoT. Single liver $\mathrm{cT}_{1}$ measurements cannot separate the individual contributions of liver fibrosis and inflammation, and presently the only method that can do this is liver biopsy. However, these results indicate that relative temporal $\mathrm{cT}_{1}$ response could differentiate between patients with cirrhosis vs non-cirrhosis and inflammation vs fibrosis reduction. Paired biopsy would be needed to confirm this but was outside the scope of this study. It also indicates that most of the improvement in fibroinflammation in patients without cirrhosis is observed by 24 weeks EoT, which is supported by the study by Bradley et al. [19] who observed significant short-term $\mathrm{T}_{1}$ changes.

Multiple studies have reported significant decreases in TE measurements and in blood-based biomarkers of fibrosis in patients undergoing DAA treatment in as little as 12 weeks and up to as long as 12 months EoT using TE [20-26, 50], in accordance with our findings. We observed a weaker correlation between liver $\mathrm{cT}_{1}$ and $\mathrm{TE}$ after treatment than prior to treatment ( $r=0.134$ vs 0.547 ). While TE correlates well with histological liver fibrosis in untreated HCV [51-53], a study has shown it to have poorer ability to identify cirrhosis in patients post-HCV treatment than in patients pretreatment [54]. Using a cut-off of $>12 \mathrm{kPa}$ for cirrhosis in that study, $21 \%$ of patients classified without cirrhosis had cirrhosis and $38 \%$ of patients with cirrhosis post-treatment were misclassified. In our study, it is likely that a significant reduction in inflammation has affected the post-treatment correlation between liver $\mathrm{cT}_{1}$ and $\mathrm{TE}$.

Liver $\mathrm{cT}_{1}$ has been shown to predict higher risk of liverrelated clinical outcomes [32], including HCC. We observed no new incidence of HCC in any DAA-treated patient; however, this was a small cohort with a relatively short followup. Alongside fibrosis and inflammation epigenetic change may also affect HCC risk post SVR. Epigenetic aberrations are linked to tumor development. Chronic HCV has been shown to induce epigenetic changes, which persist following viral eradication by DAAs $[55,56]$. It is postulated therefore that some patients, regardless of whether a reversal in either fibrosis or inflammation takes place, may still be at risk of HCC development due to persistent epigenetic alterations. Longer follow-up in larger cohorts is needed in these patients who have undergone fibroinflammatory reversal, as structural changes in the liver and residual fibroinflammation may have an influence on the prognostic value of liver $\mathrm{cT}_{1}$.

In addition to the small numbers and relatively short follow-up, the greatest limitation of this study is the lack of paired histology. However, liver fat measurement with MRS is well-validated against histology and liver $\mathrm{cT}_{1}$ has been shown to correlate well with histological fibrosis, inflammation and ballooning [29, 30]. Additionally, liver biopsy is not a true gold standard against which to measure due to sampling variation and interobserver variability $[57,58]$. Furthermore, the current staging system for fibrosis may not be optimal in the context of fibrosis regression in viral 
hepatitis [59]. Additionally, we only investigated patients with $\mathrm{TE}>8 \mathrm{kPa}$ at baseline, whereas patients with lower baseline fibroinflammation may exhibit more varied changes in liver $\mathrm{cT}_{1}$.

In this study we observed reductions in liver $\mathrm{cT}_{1}$ and liver fat following DAA therapy. Changes in both these parameters have also been observed in response to resolution of non-alcoholic steatohepatitis (NASH) [35]. This indicates that multiparametric MRI may be of use as a monitoring biomarker.

Further studies should investigate whether multiparametric MRI also changes in response to the treatment of other liver diseases (e.g., alcohol cessation in ArLD). Long term follow-up for HCC and other liver-related complications is also needed to evaluate the prognostic capability of changes in liver $\mathrm{cT}_{1}$ in patients with $\mathrm{HCV}$ after successful DAA treatment.

In conclusion, this study has shown that liver $\mathrm{cT}_{1}$ decreases after DAA treatment for chronic HCV. It is unclear to what extent these reductions are influenced by inflammation or fibrosis regression but may represent inflammatory change accompanied later by concurrent fibrosis regression.

Acknowledgements The authors would like to thank Justyna Szczurkowska for performing transient elastography and Ana Vieira, Jane Phillips and Vicki Wharton for help in patient recruitment. The views expressed in this article are those of the author are not necessarily those of the NHS, the NIHR, or the Department of Health.

Author contributions ANAJ, CL, MP, SN and EB designed the study. ANAJ, CL, JC and MP recruited patients to the study. ANAJ performed imaging and statistical analysis. ANAJ, CL, MP, MK, EB drafted the manuscript. All authors reviewed the manuscript critically and have approved the final version.

Funding ANAJ is funded by a Medical Research Council studentship. $\mathrm{CL}$ is funded by an NIHR doctoral research fellowship. SN and MP acknowledge support from the Oxford NIHR Biomedical Research Centre (BRC). EB is funded by the Medical Research Council UK, the Oxford NIHR Biomedical Research Centre and is an NIHR Senior Investigator.

Data availability Data are available from the corresponding author on reasonable request.

\section{Compliance with ethical standards}

Conflict of interest ANAJ, MP, EMT, MK, EB and SN are shareholders in Perspectum Ltd. MK is an employee of Perspectum. EMT, EB, $\mathrm{SN}$ and MP have filed patent applications related to the use of MRI for the assessment of liver disease. CL has nothing to declare.

Open Access This article is licensed under a Creative Commons Attribution 4.0 International License, which permits use, sharing, adaptation, distribution and reproduction in any medium or format, as long as you give appropriate credit to the original author(s) and the source, provide a link to the Creative Commons licence, and indicate if changes were made. The images or other third party material in this article are included in the article's Creative Commons licence, unless indicated otherwise in a credit line to the material. If material is not included in the article's Creative Commons licence and your intended use is not permitted by statutory regulation or exceeds the permitted use, you will need to obtain permission directly from the copyright holder. To view a copy of this licence, visit http://creativecommons.org/licenses/by/4.0/.

\section{References}

1. World Health Organization. Global Hepatitis Report 2017. Geneva: World Health Organization; 2017 Licence: CC BY-NCSA 3.0 IGO. WHO. 2017.

2. Ferenci P, Bourgeois S, Buggisch P, Norris S, Curescu M, Larrey $\mathrm{D}$, et al. Real-world safety and effectiveness of ombitasvir/ paritaprevir/ritonavir \pm dasabuvir \pm ribavirin in hepatitis $\mathrm{C}$ virus genotype 1 - and 4-infected patients with diverse comorbidities and comedications: A pooled analysis of post-marketing observational studi. J Viral Hepat. 2019;26(6):685-96.

3. Zeuzem S, Bourgeois S, Greenbloom S, Buti M, Aghemo A, Lampertico P, et al. JNJ-4178 (AL-335, Odalasvir, and Simeprevir) for 6 or 8 Weeks in Hepatitis C Virus-Infected Patients Without Cirrhosis: OMEGA-1. Hepatology. 2019;69(6):2349-63.

4. Fontana RJ, Lens S, McPherson S, Elkhashab M, Ankoma-Sey V, Bondin M, et al. Efficacy and Safety of 8 Weeks of Glecaprevir/ Pibrentasvir in Treatment-Naïve, HCV-Infected Patients with APRI $\leq 1$ in a Single-Arm, Open-Label, Multicenter Study. Adv Ther. 2019 Dec 23;36(12):3458-70. Available from: https://doi. org/10.1007/s12325-019-01123-0

5. De Lédinghen V, Laforest C, Hézode C, Pol S, Renault A, Alric L, et al. Retreatment with Sofosbuvir Plus Grazoprevir/Elbasvir Plus Ribavirin of Patients with Hepatitis C Virus Genotype 1 or 4 Who Previously Failed an NS5A-or NS3-Containing Regimen: The ANRS HC34 REVENGE Study. Clin Infect Dis. 2018;66(7):1013-8.

6. Llaneras J, Riveiro-Barciela M, Lens S, Diago M, Cachero A, García-Samaniego J, et al. Effectiveness and safety of sofosbuvir/ velpatasvir/voxilaprevir in patients with chronic hepatitis $\mathrm{C}$ previously treated with DAAs. J Hepatol. 2019 Oct;71(4):666-72. Available from: https://doi.org/10.1016/j.jhep.2019.06.002

7. Esteban R, Pineda JA, Calleja JL, Casado M, Rodríguez M, Turnes $\mathrm{J}$, et al. Efficacy of Sofosbuvir and Velpatasvir, With and Without Ribavirin, in Patients With Hepatitis C Virus Genotype 3 Infection and Cirrhosis. Gastroenterology. 2018;155(4):1120-1127.e4. Available from: https://linkinghub.elsevier.com/retrieve/pii/S0016 508518346845

8. Brown RS, Buti M, Rodrigues L, Chulanov V, Chuang W-L, Aguilar $\mathrm{H}$, et al. Glecaprevir/pibrentasvir for 8 weeks in treatmentnaïve patients with chronic HCV genotypes 1-6 and compensated cirrhosis: The EXPEDITION-8 trial. J Hepatol. 2019 Nov; Available from: https://doi.org/10.1016/j.jhep.2019.10.020

9. Puoti M, Foster GR, Wang S, Mutimer D, Gane E, Moreno C, et al. High SVR12 with 8-week and 12-week glecaprevir/pibrentasvir therapy: An integrated analysis of HCV genotype 1-6 patients without cirrhosis. J Hepatol. 2018 Aug;69(2):293-300. Available from: https://doi.org/10.1016/j.jhep.2018.03.007

10. Toyoda H, Atsukawa M, Watanabe T, Nakamuta M, Uojima H, Nozaki A, et al. Real-world experience of 12-week DAA regimen of glecaprevir and pibrentasvir in patients with chronic HCV infection. J Gastroenterol Hepatol. 2019 Oct 14;24(1). Available from: https://onlinelibrary.wiley.com/doi/abs/https://doi. org/10.1111/jgh.14874

11. Toyoda H, Atsukawa M, Uojima H, Nozaki A, Tamai H, Takaguchi K, et al. Trends and Efficacy of Interferon-Free Anti-hepatitis 
C Virus Therapy in the Region of High Prevalence of Elderly Patients, Cirrhosis, and Hepatocellular Carcinoma: A Real-World, Nationwide, Multicenter Study of 10688 Patients in Japan. Open Forum Infect Dis. 2019 May 1;6(5). Available from: https://acade mic.oup.com/ofid/article/doi/10.1093/ofid/ofz185/5457842

12. Foster GR, Irving WL, Cheung MCM, Walker AJ, Hudson BE, Verma $S$, et al. Impact of direct acting antiviral therapy in patients with chronic hepatitis $\mathrm{C}$ and decompensated cirrhosis. J Hepatol. 2016;64(6):1224-31. Available from: https://doi.org/10.1016/j. jhep.2016.01.029

13. Galle PR, Forner A, Llovet JM, Mazzaferro V, Piscaglia F, Raoul J-L, et al. EASL Clinical Practice Guidelines: Management of hepatocellular carcinoma. J Hepatol. 2018 Jul;69(1):182-236. Available from: https://doi.org/10.1016/j.jhep.2018.03.019

14. Piñero F, Mendizabal M, Ridruejo E, Herz Wolff F, Ameigeiras $\mathrm{B}$, Anders M, et al. Treatment with direct-acting antivirals for HCV decreases but does not eliminate the risk of hepatocellular carcinoma. Ong J, editor. Liver Int. 2019 Jun 4;39(6):1033-43. Available from: https://onlinelibrary.wiley.com/doi/abs/10.1111/ liv. 14041

15. Waziry R, Hajarizadeh B, Grebely J, Amin J, Law M, Danta M, et al. Hepatocellular carcinoma risk following direct-acting antiviral HCV therapy: A systematic review, meta-analyses, and metaregression. J Hepatol. 2017;67(6):1204-12. Available from: https ://doi.org/10.1016/j.jhep.2017.07.025

16. Nahon P, Bourcier V, Layese R, Audureau E, Cagnot C, Marcellin $\mathrm{P}$, et al. Eradication of Hepatitis C Virus Infection in Patients With Cirrhosis Reduces Risk of Liver and Non-Liver Complications. Gastroenterology. 2017 Jan;152(1):142-156.e2. Available from: http://linkinghub.elsevier.com/retrieve/pii/S0016508516350703

17. Ioannou GN, Beste LA, Green PK, Singal AG, Tapper EB, Waljee AK, et al. Increased Risk for Hepatocellular Carcinoma Persists Up to 10 Years After HCV Eradication in Patients With Baseline Cirrhosis or High FIB-4 Scores. Gastroenterology. 2019;157(5):1264-1278.e4. Available from: https://doi. org/10.1053/j.gastro.2019.07.033

18. Ioannou GN, Green PK, Beste LA, Mun EJ, Kerr KF, Berry K. Development of models estimating the risk of hepatocellular carcinoma after antiviral treatment for hepatitis C. J Hepatol. 2018;69(5):1088-98. Available from: https://doi.org/10.1016/j. jhep.2018.07.024

19. Bradley C, Scott RA, Cox E, Palaniyappan N, Thomson BJ, Ryder $\mathrm{SD}$, et al. Short-term changes observed in multiparametric liver MRI following therapy with direct-acting antivirals in chronic hepatitis C virus patients. Eur Radiol. 2019 Jun 30;29(6):31007. Available from: http://link.springer.com/10.1007/s0033 0-018-5788-1

20. Bachofner JA, Valli P V., Kröger A, Bergamin I, Künzler P, Baserga A, et al. Direct antiviral agent treatment of chronic hepatitis $\mathrm{C}$ results in rapid regression of transient elastography and fibrosis markers fibrosis-4 score and aspartate aminotransferaseplatelet ratio index. Liver Int. 2017 Mar;37(3):369-76. Available from: http://www.ncbi.nlm.nih.gov/pubmed/27678216

21. Dolmazashvili E, Abutidze A, Chkhartishvili N, Karchava M, Sharvadze L, Tsertsvadze T. Regression of liver fibrosis over a 24-week period after completing direct-acting antiviral therapy in patients with chronic hepatitis $\mathrm{C}$ receiving care within the national hepatitis $\mathrm{C}$ elimination program in Georgia: results of hepatology clinic HEPA exper. Eur J Gastroenterol Hepatol. 2017 Nov;29(11):1223-30. Available from: http://www.ncbi.nlm.nih. gov/pubmed/28857900

22. Knop V, Hoppe D, Welzel T, Vermehren J, Herrmann E, Vermehren A, et al. Regression of fibrosis and portal hypertension in $\mathrm{HCV}$-associated cirrhosis and sustained virologic response after interferon-free antiviral therapy. J Viral Hepat. 2016
Dec;23(12):994-1002. Available from: http://www.ncbi.nlm.nih. gov/pubmed/27500382

23. Chan J, Gogela N, Zheng H, Lammert S, Ajayi T, Fricker Z, et al. Direct-Acting Antiviral Therapy for Chronic HCV Infection Results in Liver Stiffness Regression Over 12 Months Posttreatment. Dig Dis Sci. 2018 Feb 8;63(2):486-92. Available from: http://link.springer.com/10.1007/s10620-017-4749-x

24. Elsharkawy A, Alem SA, Fouad R, El Raziky M, El Akel W, Abdo $\mathrm{M}$, et al. Changes in liver stiffness measurements and fibrosis scores following sofosbuvir based treatment regimens without interferon. J Gastroenterol Hepatol. 2017;32(9):1624-30.

25. Laursen TL, Siggaard CB, Kazankov K, Sandahl TD, Møller HJ, Tarp B, et al. Time-dependent improvement of liver inflammation, fibrosis and metabolic liver function after successful direct-acting antiviral therapy of chronic hepatitis C. J Viral Hepat. 2020 Jan 2;27(1):28-35. Available from: https://onlin elibrary.wiley.com/doi/abs/10.1111/jvh.13204.

26. Stasi C, Sadalla S, Carradori E, Monti M, Petraccia L, Madia $\mathrm{F}$, et al. Longitudinal evaluation of liver stiffness and outcomes in patients with chronic hepatitis $\mathrm{C}$ before and after short- and long-term IFN-free antiviral treatment. Curr Med Res Opin. 2019 Nov 8;0(0):1-1. Available from: https://doi. org/10.1080/03007995.2019.1691517

27. Pan JJ, Bao F, Du E, Skillin C, Frenette CT, Waalen J, et al. Morphometry Confirms Fibrosis Regression From Sustained Virologic Response to Direct-Acting Antivirals for Hepatitis C. Hepatol Commun. 2018;2(11):1320-30.

28. Tunnicliffe EM, Banerjee R, Pavlides M, Neubauer S, Robson MD. A model for hepatic fibrosis: the competing effects of cell loss and iron on shortened modified Look-Locker inversion recovery T1(shMOLLI-T1) in the liver. J Magn Reson Imaging. 2017;45(2):450-62.

29. Banerjee R, Pavlides M, Tunnicliffe EM, Piechnik SK, Sarania N, Philips R, et al. Multiparametric magnetic resonance for the non-invasive diagnosis of liver disease. J Hepatol. 2014 Jan 7;60(1):69-77. Available from: https://doi.org/10.1016/j. jhep.2013.09.002

30. Pavlides M, Banerjee R, Tunnicliffe EM, Kelly C, Collier J, Wang LM, et al. Multiparametric magnetic resonance imaging for the assessment of non-alcoholic fatty liver disease severity. Liver Int. 2017 Jul;37(7):1065-73. Available from: http://doi. wiley.com/10.1111/liv.13284

31. Bachtiar V, Kelly MD, Wilman HR, Jacobs J, Newbould R, Kelly CJ, et al. Repeatability and reproducibility of multiparametric magnetic resonance imaging of the liver. Lundberg $\mathrm{P}$, editor. PLoS One. 2019 Apr 10;14(4):e0214921. Available from: http://dx.plos.org/10.1371/journal.pone.0214921

32. Pavlides M, Banerjee R, Sellwood J, Kelly CJ, Robson MD, Booth JC, et al. Multiparametric magnetic resonance imaging predicts clinical outcomes in patients with chronic liver disease. J Hepatol. 2016 Feb;64(2):308-15. Available from: https://doi. org/10.1016/j.jhep.2015.10.009.

33. Ryan JD, Armitage AE, Cobbold JF, Banerjee R, Borsani $\mathrm{O}$, Dongiovanni $\mathrm{P}$, et al. Hepatic iron is the major determinant of serum ferritin in NAFLD patients. Liver Int. 2018;38(1):164-73..

34. McPherson S, Jonsson JR, Cowin GJ, O'Rourke P, Clouston AD, Volp A, et al. Magnetic resonance imaging and spectroscopy accurately estimate the severity of steatosis provided the stage of fibrosis is considered. J Hepatol. 2009 Aug;51(2):389-97. Available from: https://linkinghub.elsevier.com/retrieve/pii/S016882780 9003171

35. Harrison SA, Rossi SJ, Paredes AH, Trotter JF, Bashir MR, Guy CD, et al. NGM282 Improves Liver Fibrosis and Histology in 12 Weeks in Patients With Nonalcoholic Steatohepatitis. Hepatology. 
2019 Apr 10;0(0):1-15. Available from: https://onlinelibrary.wiley .com/doi/abs/10.1002/hep.30590

36. Purvis LAB, Clarke WT, Biasiolli L, Valkovič L, Robson MD, Rodgers CT. OXSA: An open-source magnetic resonance spectroscopy analysis toolbox in MATLAB. PLoS One. 2017;12(9).

37. Vanhamme L, van den Boogaart A, Van Huffel S. Improved Method for Accurate and Efficient Quantification of MRS Data with Use of Prior Knowledge. J Magn Reson. 1997;129(1):35-43.

38. Rial B, Robson MD, Neubauer S, Schneider JE. Rapid quantification of myocardial lipid content in humans using single breathhold 1H MRS at 3 Tesla. Magn Reson Med. 2011;66:619-24.

39. Sterling RK, Lissen E, Clumeck N, Sola R, Correa MC, Montaner $\mathrm{J}$, et al. Development of a simple noninvasive index to predict significant fibrosis in patients with HIV/HCV coinfection. Hepatology. 2006 Jun;43(6):1317-25. Available from: http://doi.wiley .com/10.1002/hep.21178.

40. Wai CT, Greenson JK, Fontana RJ, Kalbfleisch JD, Marrero JA, Conjeevaram HS, et al. A simple noninvasive index can predict both significant fibrosis and cirrhosis in patients with chronic hepatitis C. Hepatology. 2003;38(2):518-26.

41. Mozes FE, Tunnicliffe EM, Pavlides M, Robson MD. Influence of fat on liver T1 measurements using modified Look-Locker inversion recovery (MOLLI) methods at 3T. J Magn Reson Imaging. 2016 Jul;44(1):105-11. Available from: http://www.ncbi.nlm.nih. gov/pubmed/26762615

42. R Core Team. R: A language and environment for statistical computing [Internet]. R Foundation for Statistical Computing, Vienna, Austria; 2018. Available from: https://www.r-project.org/

43. Harrison SA, Dennis A, Fiore MM, Kelly MD, Kelly CJ, Paredes $\mathrm{AH}$, et al. Utility and variability of three non-invasive liver fibrosis imaging modalities to evaluate efficacy of GR-MD-02 in subjects with NASH and bridging fibrosis during a phase-2 randomized clinical trial. Huang J-F, editor. PLoS One. 2018 Sep 7;13(9):e0203054. Available from: http://dx.plos.org/10.1371/ journal.pone. 0203054

44. Poynard T, Moussalli J, Munteanu M, Thabut D, Lebray P, Rudler $\mathrm{M}$, et al. Slow regression of liver fibrosis presumed by repeated biomarkers after virological cure in patients with chronic hepatitis C. J Hepatol. 2013 Oct;59(4):675-83. Available from: https://doi. org/10.1016/j.jhep.2013.05.015

45. Pekow JR, Bhan AK, Zheng H, Chung RT. Hepatic steatosis is associated with increased frequency of hepatocellular carcinoma in patients with hepatitis C-related cirrhosis. Cancer. 2007 Jun 15;109(12):2490-6. Available from: http://doi.wiley.com/10.1002/ cncr.22701

46. Hasan Y, Brown K. Viral eradication restores normal iron status in chronic hepatitis $\mathrm{C}$ patients with abnormal iron studies. Ann Hepatol. 2020 Mar; Available from: https://linkinghub.elsevier. com/retrieve/pii/S1665268120300247.

47. Ganne-Carrié N, Christidis C, Chastang C, Ziol M, Chapel F, Imbert-Bismut $\mathrm{F}$, et al. Liver iron is predictive of death in alcoholic cirrhosis: A multivariate study of 229 consecutive patients with alcoholic and/or hepatitis c virus cirrhosis: A prospective follow up study. Gut. 2000;46(2):277-82.

48. Nuttall KL, Palaty J, Lockitch G. Reference limits for copper and iron in liver biopsies. Ann Clin Lab Sci. 2003;33(4):443-50. Available from: http://www.ncbi.nlm.nih.gov/pubmed/14584759

49. Ioannou GN, Dominitz JA, Weiss NS, Heagerty PJ, Kowdley K $\mathrm{V}$. The effect of alcohol consumption on the prevalence of iron overload, iron deficiency, and iron deficiency anemia. Gastroenterology. 2004 May;126(5):1293-301. Available from: https://linki nghub.elsevier.com/retrieve/pii/S0016508504000940

50. Knop V, Hoppe D, Welzel T, Vermehren J, Herrmann E, Vermehren A, et al. Regression of fibrosis and portal hypertension in $\mathrm{HCV}$-associated cirrhosis and sustained virologic response after interferon-free antiviral therapy. J Viral Hepat. 2016 Dec;23(12):994-1002. Available from: http://doi.wiley .com/10.1111/jvh.12578

51. Ziol M, Handra-Luca A, Kettaneh A, Christidis C, Mal F, Kazemi $\mathrm{F}$, et al. Noninvasive assessment of liver fibrosis by measurement of stiffness in patients with chronic hepatitis C. Hepatology. 2005;41(1):48-54.

52. Sandrin L, Fourquet B, Hasquenoph JM, Yon S, Fournier $\mathrm{C}$, Mal F, et al. Transient elastography: A new noninvasive method for assessment of hepatic fibrosis. Ultrasound Med Biol. 2003;29(12):1705-13.

53. Castéra L, Vergniol J, Foucher J, Le Bail B, Chanteloup E, Haaser $\mathrm{M}$, et al. Prospective comparison of transient elastography, Fibrotest, APRI, and liver biopsy for the assessment of fibrosis in chronic hepatitis C. Gastroenterology. 2005;128(2):343-50.

54. D'Ambrosio R, Aghemo A, Fraquelli M, Rumi MG, Donato MF, Paradis V, et al. The diagnostic accuracy of Fibroscan ${ }^{\circledR}$ for cirrhosis is influenced by liver morphometry in HCV patients with a sustained virological response. J Hepatol. 2013 Aug;59(2):251-6. Available from: https://doi.org/10.1016/j.jhep.2013.03.013

55. Hamdane N, Jühling F, Crouchet E, El Saghire H, Thumann C, Oudot MA, et al. HCV-Induced Epigenetic Changes Associated With Liver Cancer Risk Persist After Sustained Virologic Response. Gastroenterology. 2019;156(8):2313-2329.e7. Available from: https://doi.org/10.1053/j.gastro.2019.02.038

56. Perez S, Kaspi A, Domovitz T, Davidovich A, Lavi-Itzkovitz A, Meirson $\mathrm{T}$, et al. Hepatitis $\mathrm{C}$ virus leaves an epigenetic signature post cure of infection by direct-acting antivirals. Rowe HM, editor. PLOS Genet. 2019 Jun 19;15(6):e1008181. Available from: http:// dx.plos.org/10.1371/journal.pgen.1008181

57. Gronbaek K, Christensen PB, Hamilton-Dutoit S, Federspiel BH, Hage E, Jensen OJ, et al. Interobserver variation in interpretation of serial liver biopsies from patients with chronic hepatitis C. J Viral Hepat. 2002 Nov 13;9(6):443-9. Available from: https://doi. org/10.1046/j.1365-2893.2002.00389.x

58. Robert M, Sofair AN, Thomas A, Bell B, Bialek S, Corless C, et al. A Comparison of Hepatopathologists' and Community Pathologists' Review of Liver Biopsy Specimens From Patients With Hepatitis C. Clin Gastroenterol Hepatol. 2009 Mar;7(3):335-8. Available from: https://linkinghub.elsevier.com/ retrieve/pii/S1542356508012317

59. Sun Y, Zhou J, Wang L, Wu X, Chen Y, Piao H, et al. New classification of liver biopsy assessment for fibrosis in chronic hepatitis B patients before and after treatment. Hepatology. 2017;65(5):1438-50.

Publisher's Note Springer Nature remains neutral with regard to jurisdictional claims in published maps and institutional affiliations. 